DRAFT VERSION NOVEMBER 5, 2018

Preprint typeset using LTEX style emulateapj v. 8/13/10

\title{
THE PROGENITOR MASS OF SN 2011DH FROM STELLAR POPULATIONS ANALYSIS
}

\author{
Jeremiah W. Murphy ${ }^{1,2}$, Zachary G. Jennings ${ }^{1}$, Benjamin Williams ${ }^{1}$, Julianne J. DalCANTON ${ }^{1}$, AND ANDreW E. Dolphin ${ }^{3}$
}

Draft version November 5, 2018

\begin{abstract}
Using Hubble Space Telescope (HST) photometry, we characterize the age of the stellar association in the vicinity of supernova (SN) $2011 \mathrm{dh}$ and use it to infer the zero-age main sequence mass ( $M_{\text {ZAMS }}$ ) of the progenitor star. We find two distinct and significant star formation events with ages of $<6$ and $17_{-4}^{+3}$ Myrs, and the corresponding $M_{\text {ZAMS }}$ are $>29$ and $13_{-1}^{+2} \mathrm{M}_{\odot}$, respectively. These two bursts represent $18_{-9}^{+4} \%$ (young) and $64_{-14}^{+10} \%$ (old) of the total star formation in the last 50 Myrs. Adopting these fractions as probabilities suggests that the most probable $M_{\text {ZAMS }}$ is $13_{-1}^{+2} \mathrm{M}_{\odot}$. These results are most sensitive to the luminosity function along the well-understood main sequence and are less sensitive to uncertain late-stage stellar evolution. Therefore, they stand even if the progenitor suffered disruptive post-main-sequence evolution (e.g. eruptive mass loss or binary Roche-lobe overflow). Progenitor identification will help to further constrain the appropriate population. Even though pre-explosion images show a yellow supergiant (YSG) at the site of the SN, panchromatic SN light curves suggest a more compact star as the progenitor. In spite of this, our results suggest an association between the YSG and the SN. Not only was the star located at the SN site, but reinforcing an association, the star's bolometric luminosity is consistent with the final evolutionary stage of the $17 \mathrm{Myr}$ old star burst. If the YSG disappears, then $M_{\text {ZAMS }}=13_{-1}^{+2} \mathrm{M}_{\odot}$, but if it persists, then our results allow the possibility that the progenitor was an unseen star of $>29 \mathrm{M}_{\odot}$.
\end{abstract}

Subject headings: supernovae: general — supernovae: individual (SN 2011dh)

\section{INTRODUCTION}

Observational measurements of the masses of supernova (SN) progenitors are currently scant and highly uncertain. Of the $\sim 20 \mathrm{SNe}$ that have progenitor mass constraints, only about half have well-defined masses and the rest have upper bounds (Arnett et al. 1989; Aldering et al. 1994; Barth et al. 1996; Van Dyk et al. 1999, 2002; Smartt et al. 2002; Van Dyk et al. 2003a b; Smartt et al. 2004; Maund et al. 2005; Hendry et al. 2006; Li et al. 2005, 2006, 2007; Gal-Yam et al. 2007; Crockett et al. 2008; Gal-Yam \& Leonard 2009; Smartt et al. 2009; Smartt 2009; Smith et al. 2011a b). In general, stellar evolution theory predicts a clear mapping between the zero-age main sequence mass ( $M_{\text {ZAMS }}$ ) and the explosion scenario for isolated massive stars Woosley et al. 2002; Heger et al. 2003; Dessart et al. 2011). These limited observations suggest that the least massive stars explode as SN II-P as expected (Smartt et al. 2009). More massive stars are expected to lose much of their mass and explode as H-deficient $\mathrm{SNe}$ (IIb and Ib/c). However, some H-rich SNe (in particular IIn) have been associated with very massive stars (Gal-Yam \& Leonard 2009; Smith et al. 2011ab). Furthermore, theory (Claeys et al. 2011; Dessart et al. 2011) and the relatively high observed rates of H-deficient SNe (Smith et al. 2011b) imply that binary evolution may figure prominently in producing the $\mathrm{H}$ deficient SNe. Even in light of these complications, the maximum $M_{\text {ZAMS }}$ associated with SN II-P is much lower than expected (Smartt et al. 2009). While tantalizing, these initial results are poorly constrained, and even the simple statement

\footnotetext{
${ }^{1}$ Astronomy Department, The University of Washington Seattle, WA 98195; jmurphy@astro.washington.edu

2 NSF Astronomy and Astrophysics Postdoctoral Fellow

${ }^{3}$ Raytheon, 1151 E. Hermans Road, Tucson, AZ 85706; adolphin@raytheon.com
}

that stars more massive than $\sim 8 \mathrm{M}_{\odot}$ explode as $\mathrm{SNe}$ requires more observational constraints. Thus every new supernova marks an important opportunity to add a new progenitor mass measurement to this poorly-constrained sample.

Recently, amateurs and the Palomar Transient Factory (PTF) collaboration detected supernova (SN) 2011dh in M51, the Whirlpool Galaxy (Griga et al. 2011; Silverman et al. 2011; Arcavi et al. 2011). Initial reports classified it as II (Silverman et al. 2011), but late time spectra reveals this explosion to belong to the rare IIb transitional class (Arcavi et al. 2011; Marion et al. 2011), indicating that the progenitor lost much of its hydrogen envelope due to either binary evolution or unknown single-star mass-loss physics. The close proximity of this $\mathrm{SN}\left((M-m)_{0}=29.42,7.7 \mathrm{Mpc}\right.$; Tonry et al. 2001, companion galaxy NGC 5195) and the wealth of Hubble Space Telescope (HST) data on M51 offers a rare opportunity to further explore the physics of the progenitor.

Using archival HST photometry, Maund et al. (2011) and Van Dyk et al. (2011) identified a yellow supergiant (YSG) as the progenitor candidate. Van Dyk et al. (2011) fit the magnitude and color of the progenitor to evolutionary tracks and derive a zero-age-main-sequence progenitor mass of $M_{\mathrm{ZAMS}}=$ 18-21 $\mathrm{M}_{\odot}$. However, Maund et al. (2011) argue that an uncertain mass-loss history causes the color to be an unreliable characteristic of the progenitor. Instead, they treat the bolometric luminosity as an intrinsic property determined by the core mass, which is in turn determined by MZAMs. Matching the bolometric luminosity to the very last stages of evolutionary models, they derive $M_{\mathrm{ZAMS}}=13 \pm 3 \mathrm{M}_{\odot}$. To further complicate the situation, recent observations suggest that the YSG may not even be the progenitor. Based upon the characteristics of the SN light curves, Arcavi et al. (2011) and Soderberg et al. (2011) argue that the YSG is not the progenitor and instead suggest a more compact source. 
This discrepancy in mass measurements highlights the fact that interpretation of the precursor photometry alone is sensitive to uncertain mass-loss physics in the final evolutionary stages of the models. Furthermore, using precursor imaging to measure progenitor masses requires that precursor imaging exists and that the $\mathrm{SN}$ position is known to sub-arcsecond precision. The majority of past $\mathrm{SNe}$ have neither pre-existing HST imaging, nor sufficiently accurate astrometry. A significant fraction of future nearby SNe will lack precursor imaging as well.

In this letter, we address the progenitor mass with an independent approach; we characterize the age of the stellar association surrounding SN 2011dh and derive the corresponding $M_{\text {ZAMS }}$ for the progenitor. This technique was thoroughly described by Gogarten et al. (2009) on a SN "impostor" in NGC 300 and by Badenes et al. (2009) on SN remnants in the Magellanic Clouds. In brief, using well-established stellar population modeling techniques, we can age-date the star formation episode that led to the observed SN. The resulting age can place strong constraints on the mass of the precursor by leveraging the well-understood properties of a large number of main-sequence stars. This complementary approach is feasible even when there is no precursor imaging, or when the SN position is only localized to within several arcseconds.

In $\S 2$, we detail our application of this technique to SN $2011 \mathrm{dh}$, the most distant and only ongoing SN to which it has been applied. In $\S 3$, we provide the resulting age and mass measurements, and in $\$ 4$, we compare our resulting progenitor mass with those obtained using direct imaging of the progenitor (Maund et al. 2011; Van Dyk et al. 2011). We find our results to be most consistent with the results of (Maund et al. 2011) and improve upon their uncertainties.

\section{DATA \& ANALYSIS}

\subsection{Data Acquisition and Photometry}

The region surrounding SN 2011dh was imaged with HST using the Advanced Camera for Surveys (ACS) on January 20-21, 2005 (Hubble Heritage Team, target name M51-POS5, Proposal 10452). For our analysis, we retrieved calibrated data from the HST Archive in the deepest two of the four available filters, F555W and F814W. Four dithered images of exposure time $340 \mathrm{~s}$ were available in each filter, providing a total exposure time of $1360 \mathrm{~s}$ in each band.

Resolved stellar photometry was performed using the photometry pipeline developed for the ACS Nearby Galaxy Treasury program (Dalcanton et al. 2009). This pipeline uses the DOLPHOT stellar photometry package (Dolphin 2000) to fit the ACS point spread function (PSF) to all of the point sources in the images. Fluxes are then converted to Vega magnitudes using the standard zero-points and aperture corrections from the ACS handbook. To assess photometric errors and completeness, at least $10^{5}$ fake star tests are performed by inserting fake stars of known color and magnitude into the data and blindly attempting to recover them with the same software. For details of the quality cuts, see Dalcanton et al. (2009).

\subsection{Recent Star Formation History Recovery}

For our analysis, we selected stars within $1.4^{\prime \prime}(\sim 50 \mathrm{pc})$ of the SN location (Gogarten et al. 2009). By varying the radius from 30 to $100 \mathrm{pc}$, we verify that $50 \mathrm{pc}$ maximizes the SF in the local bursts and minimizes contamination. This resulted in 29 stars being selected, including 16 upper main sequence stars. A color-magnitude diagram (CMD) of these stars is displayed in the left panel of Fig. 1.
We fit the stellar evolution models of Girardi et al. 2002, 2010) to our data using the software package MATCH, which calculates a maximum likelihood fit of a linear combination of model CMDs to the observed CMD (Dolphin 2002). We verified that our CMD was consistent with a population located at the published distance of M51 $\left((m-M)_{0}=29.42\right.$, Tonry et al. 2001) by allowing MATCH to fit the distance modulus. The best-fit distance modulus is $(m-M)_{0}=29.45$ and is consistent with the published distance. MATCH also fits for a distribution of extinctions; we fix the width of the distribution at 0.5 , but allow the minimum to vary from $A v=0.0$ to 0.5 . We constrained the metallicity to increase for younger ages. We only fit data brighter than our $50 \%$ completeness limit as measured by our artificial star tests. These limits were $m<26.95$ in both filters.

Having calculated a best-fit age distribution, we quantify the random and systematic uncertainties. The random error is due to sampling statistics and is largely determined by the number of stars in the sample. The systematic error is due to potential offsets between model and observed CMDs. For example, if the model is systematically slightly bluer than the data (potentially due to systematic offsets in the model transformations or model deficiencies), then the resulting age distribution will be affected. We estimate the uncertainty from both of these sources through a series of Monte Carlo (MC) realizations of the data. Each realization draws the same number of stars as our data from the best-fit age distribution. These random draws are re-fit with the models after shifting the models by 0.4 magnitudes in bolometric luminosity and $7 \%$ in effective temperature, which represent distance and systematic model uncertainties. Thus, the set of resulting fits provide a distribution of measurements from which we calculate our uncertainty. Finally, to assess the dependence of the result on individual outliers, we performed jackknifing tests in which the brightest or bluest star in the field (such as the SN progenitor candidate) was removed from the data.

Our goal in this analysis is to identify the burst of star formation in which the progenitor star was most likely created. We therefore focus on star formation history ( $\mathrm{SFH}$ ) of the past 50 Myr. To identify recent star formation bursts, we examine the cumulative star formation as a fraction of the total stellar mass formed in the past $50 \mathrm{Myr}$. We then calculate the median age of the recently formed stars as well as the width of their age distribution. This method has the advantage of only being sensitive to relative star formation rates, not absolute values. Furthermore, the cumulative distribution is less affected by the covariances between neighboring age bins in the model fits.

This methodology leads to two sources of uncertainty in age. One is the uncertainty in the median age, which the MC tests address, and the second is the width of the distribution of ages. To account for the uncertainty due to the intrinsic age, we assign probabilities to each age based on the mass fraction of stars with that age. Uncertainties on these probabilities then account for the measurement errors. Our quoted mass values correspond to the most massive star for the age limits, which are calculated directly from the same models as used for fitting (Girardi et al. 2010).

\section{RESULTS}

In this section, we report the best-fit metallicity, average extinction, and SFH of the region surrounding the location of SN 2011dh. In Fig. 2, we show the primary result, the age distribution of young populations surrounding SN 2011dh. The 
best-fit metallicity for the last $50 \mathrm{Myr}$ is solar, which compares favorably with the range of metallicities derived from HII regions in M51 (Bresolin et al. 2004). For the average extinction, we find $A_{V}=0.425$. The value for galactic extinction at this pointing is $A_{V}=0.12$ (Schlegel et al. 1998); therefore this region likely contains additional extinction from dust within M51. To estimate the uncertainty due to extinction, we force $A_{v}=0.12$ in MATCH and compare these results to the self-consistent fit. The resulting differences in ages and masses are included in the uncertainties that we report in this letter.

The left panel of Fig. 1 shows the magnitude (F555W) and color (F555W-F814W) of the brightest stars surrounding the SN, which we use to calculate the SFH in the vicinity of SN 2011dh. The green star shows the magnitude and color of the progenitor candidate, a YSG (Maund et al. 2011; Van Dyk et al. 2011). For comparison, we plot three isochrones corresponding to the three star formation (SF) events in Fig. 2. In addition, we show the corresponding age and fraction of total SF in the last 50 Myrs. The right panel of Fig. 1 shows a model CMD produced by MATCH for the best-fit SFH, with the same isochrones over-plotted. Note that the model generically reproduces the number density of stars on the MS and red giant regions of the CMD.

To be clear, we did not fit the main sequence turn-off (MTO) to arrive at these isochrones. Because of the small number of main sequence stars at the bright end, MTO fitting is susceptible to large Poisson errors. Rather, as we explain in $\S 2$, we model the entire CMD and constrain the SFH that is required to reproduce the observed CMD. This way the number density of stars at each color and magnitude, particularly along the entire MS, is involved in constraining the age of the star burst, not just the location of the end of the MS.

The best fit SFH is shown in Fig. 2. The left panel shows the relative SFH (black solid line) as a function of age, and the right panel shows the cumulative SF (thick solid purple line) since $50 \mathrm{Myr}$ ago. The orange thin lines show the $68 \%$ confidence interval for the cumulative distribution. In both plots, three bursts of SF are apparent: 4, 8, and $17 \mathrm{Myr}$. The 17 Myr burst accounts for most $\left(64_{-14}^{+10} \%\right)$ of the recent SF in the last 50 Myr.

Based on these bursts, we report two estimates for the age and $M_{\text {ZAMS }}$. The age is either $<6$ or $17_{-4}^{+3} \mathrm{Myr}$, corresponding to $M_{\text {ZAMS }}$ of either $>29$ or $13_{-1}^{+2} \mathrm{M}_{\odot}$, respectively. Since most of the SF in the last $50 \mathrm{Myr}$ is associated with the $\sim 17 \mathrm{Myr}$ burst, we find the most probable $M_{\text {ZAMS }}$ of the progenitor to be $13_{-1}^{+2} \mathrm{M}_{\odot}$. We rule out the $\sim 8 \mathrm{Myr}$ SF event for three interrelated reasons. For one, this event has uncertainties consistent with zero star formation. Secondly, jackknifing tests show that this event is strongly dependent on the presence of the YSG, suggesting that no other stars in the sample are consistent with this age. Thirdly, if the YSG is the progenitor, then its color implies that it experienced a great deal of mass-loss that is not included in the models used in MATCH. Hence, any fits that rely on the magnitude and color of this star alone are in error. See $\S 4$ for further discussion on these last two points. In the cumulative plot, the age and mass estimate associated with the younger event is highlighted by the blue shaded regions, while the older and most probable estimate is highlighted by the red shaded region.

\section{DISCUSSION \& CONCLUSIONS}

Using HST photometry, we characterize the age of the stars in the vicinity of SN $2011 \mathrm{dh}$, and from this age, we infer the $M_{\text {ZAMS }}$ of the progenitor star. The recent SFH shows two SF events with ages of $<6$ and $17_{-4}^{+3}$ Myrs, corresponding to $M_{\text {ZAMS }}$ for the progenitor of $>29$ and $13_{-1}^{+2} \mathrm{M}_{\odot}$, respectively. Given that the older burst at $17_{-4}^{+3}$ Myr represents $64_{-14}^{+10} \%$ of the total SF in the last 50 Myrs, the most probable $M_{\text {ZAMS }}$ for the progenitor is $13_{-1}^{+2} \mathrm{M}_{\odot}$. Because MATCH leverages the entire CMD, including the MS, these $M_{\text {ZAMS }}$ estimates are relatively insensitive to the individual peculiarities of post-MS or binary evolution.

Stellar population analysis shows that the colors of the YSG are consistent with the surrounding stars only if it experienced peculiar evolution. Testing the robustness of the three SF events, we perform several jackknifing tests, removing a bright star and finding new best-fit SFHs. The $<6$ and $17 \mathrm{Myr}$ old populations are robust to these tests, but upon removing the YSG, the 8 Myr old burst vanishes $(<3.5 \%$ of the recent $\mathrm{SF})$. Hence, the $8 \mathrm{Myr}$ feature is solely dependent upon the color and magnitude of the most poorly modeled star in the field, the YSG - an unlikely scenario. More likely, the YSG was born in one of the other events and experienced disruptive mass loss, giving it peculiar colors. In fact, its bolometric luminosity is consistent with the final evolutionary stage of the 17 Myr old population.

Regardless of an association with the SN, the luminosity of the YSG (Maund et al. 2011; Van Dyk et al. 2011) suggests that it is associated with the 17 Myr population, in which case, we derive a $M_{\text {ZAMS }}$ for the YSG of $13_{-1}^{+2} \mathrm{M}_{\odot}$. The previous $M_{\text {ZAMS }}$ estimates were obtained by comparing photometry of the YSG with stellar evolution models. Of the two previous attempts to estimate $M_{\text {ZAMS }}$ one at $13 \pm 3 \mathrm{M}_{\odot}$ (Maund et al. 2011) and one at 18-21 $\mathrm{M}_{\odot}$ (Van Dyk et al. 2011), the lower mass estimate is most consistent with our results. This consistency leads to a couple of conclusions.

For one, this consistency validates using nearby stellar ages to estimate the progenitor mass when an archival HST image of the progenitor is not available. Given the distance to M51, $7.7 \mathrm{Mpc}$, the fact that this technique is able to further constrain the $M_{\text {ZAMS }}$ estimate is remarkable. Directly modeling the progenitor in precursor imaging only requires accurate photometry for the most luminous stars and has become a routine exercise up to $20 \mathrm{Mpc}$ (Smartt 2009). Hence, for direct imaging of the precursor, SN 2011dh is quite close. Our technique, on the other hand, relies on having a sufficient number of main sequence stars to characterize the age of the associated star burst. With a distance of $\sim 7.7 \mathrm{Mpc}$, the magnitude limit is quite high, resulting in only 16 detectable upper main sequence stars within $50 \mathrm{pc}$ of the SN. Even with this small number of upper main sequence stars, we were able to improve upon the direct imaging mass constraint for the YSG.

The second conclusion we draw from the consistency is that fitting the bolometric luminosity of the progenitor to the last stages of evolutionary tracks (Maund et al.2011) is a more robust method to estimate $M_{\text {ZAMS }}$ than fitting both the luminosity and effective temperature (Van Dyk et al. 2011). The literature is full of theoretical arguments suggesting that the bolometric luminosity during the last evolutionary stages is a more intrinsic property of $M_{\text {ZAMS }}$ Arnett et al.1989; Woosley et al. 2002; Smartt 2009), but as far as we know, there have been no independent observations to support this. Because age-dating the nearby stars leverages information from the MS phase, our results provide a complimentary estimate of $M_{\text {ZAMS }}$ and inde- 
pendent support for using the bolometric luminosity only in direct imaging techniques.

While the most probable $M_{\text {ZAMS }}$ of the SN progenitor is $13_{-1}^{+2} \mathrm{M}_{\odot}$, there is a small but not insignificant probability that the progenitor mass is $>29 \mathrm{M}_{\odot}$. Ruling out one or the other as the progenitor requires further information. If the YSG persists in post-SN imaging, then the progenitor was indeed compact (Arcavi et al. 2011; Soderberg et al. 2011), and neither mass estimate is ruled out. However, if the YSG disappears, then our progenitor mass estimate of $13_{-1}^{+2} \mathrm{M}_{\odot}$ would validate and further constrain the Maund et al. (2011) estimate. Given the YSG's positional coincidence with the $\mathrm{SN}$ and the fact that its bolometric luminosity is consistent with the final evolutionary stage of the 17 Myr old population, it would be odd if the YSG is not associated with the SN.

We thank George Wallerstein for drawing our attention to this SN. J.W.M. is supported by an NSF Astronomy and Astrophysics Postdoctoral Fellowship under award AST0802315. Z.G.J. is supported by the same award. This work is based on observations made with the NASA/ESA Hubble Space Telescope, obtained from the data archive at the Space Telescope Science Institute. STScI is operated by the Association of Universities for Research in Astronomy, Inc. under NASA contract NAS 5-26555.

\section{REFERENCES}

Aldering, G., Humphreys, R. M., \& Richmond, M. 1994, AJ, 107, 662 Arcavi, I., Gal-Yam, A., Yaron, O., Sternberg, A., Rabinak, I., Waxman, E., Kasliwal, M. M., Quimby, R. M., Ofek, E. O., Horesh, A., Kulkarni, S. R., Filippenko, A. V., Silverman, J. M., Cenko, S. B., Li, W., Bloom, J. S., Sullivan, M., Fox, D. B., Nugent, P. E., Poznanski, D., Gorbikov, E., Riou, A., Lamotte-Bailey, S., Griga, T., Cohen, J. G., Polishook, D., Xu, D., Ben-Ami, S., Manulis, I., Walker, E. S., Mazzali, P. A., Pian, E., Matheson, T., Maquire, K., Pan, Y.-C., Bersier, D., James, P., Marchant, J. M., Smith, R. J., Mottram, C. J., Barnsley, R. M., Kandrashoff, M. T., \& Clubb, K. I. 2011, ArXiv e-prints

Arnett, W. D., Bahcall, J. N., Kirshner, R. P., \& Woosley, S. E. 1989, ARA\&A, 27, 629

Badenes, C., Harris, J., Zaritsky, D., \& Prieto, J. L. 2009, ApJ, 700, 727

Barth, A. J., van Dyk, S. D., Filippenko, A. V., Leibundgut, B., \& Richmond, M. W. 1996, AJ, 111, 2047

Bresolin, F., Garnett, D. R., \& Kennicutt, Jr., R. C. 2004, ApJ, 615, 228

Claeys, J. S. W., de Mink, S. E., Pols, O. R., Eldridge, J. J., \& Baes, M. 2011, A\&A, 528, A131+

Crockett, R. M., Eldridge, J. J., Smartt, S. J., Pastorello, A., Gal-Yam, A., Fox, D. B., Leonard, D. C., Kasliwal, M. M., Mattila, S., Maund, J. R., Stephens, A. W., \& Danziger, I. J. 2008, MNRAS, 391, L5

Dalcanton, J. J., Williams, B. F., Seth, A. C., Dolphin, A., Holtzman, J., Rosema, K., Skillman, E. D., Cole, A., Girardi, L., Gogarten, S. M., Karachentsev, I. D., Olsen, K., Weisz, D., Christensen, C., Freeman, K., Gilbert, K., Gallart, C., Harris, J., Hodge, P., de Jong, R. S.,

Karachentseva, V., Mateo, M., Stetson, P. B., Tavarez, M., Zaritsky, D., Governato, F., \& Quinn, T. 2009, ApJS, 183, 67

Dessart, L., Hillier, D. J., Livne, E., Yoon, S.-C., Woosley, S., Waldman, R., \& Langer, N. 2011, MNRAS, 414, 2985

Dolphin, A. E. 2000, PASP, 112, 1383

-. 2002, MNRAS, 332, 91

Gal-Yam, A., \& Leonard, D. C. 2009, Nature, 458, 865

Gal-Yam, A., Leonard, D. C., Fox, D. B., Cenko, S. B., Soderberg, A. M. Moon, D.-S., Sand, D. J., Li, W., Filippenko, A. V., Aldering, G., \& Copin, Y. 2007, ApJ, 656, 372
Girardi, L., Bertelli, G., Bressan, A., Chiosi, C., Groenewegen, M. A. T., Marigo, P., Salasnich, B., \& Weiss, A. 2002, A\&A, 391, 195

Girardi, L., Williams, B. F., Gilbert, K. M., Rosenfield, P., Dalcanton, J. J., Marigo, P., Boyer, M. L., Dolphin, A., Weisz, D. R., Melbourne, J., Olsen, K. A. G., Seth, A. C., \& Skillman, E. 2010, ApJ, 724, 1030

Gogarten, S. M., Dalcanton, J. J., Murphy, J. W., Williams, B. F., Gilbert, K., \& Dolphin, A. 2009, ApJ, 703, 300

Griga, T., Marulla, A., Grenier, A., Sun, G., Gao, X., Lamotte Bailey, S., Koff, R. A., Mikuz, H., Dintinjana, B., Silverman, J. M., Cenko, S. B., Filippenko, A. V., Li, W., Yamanaka, M., Itoh, R., Arai, A., Nagashima, M., \& Kajiawa, K. 2011, Central Bureau Electronic Telegrams, 2736, 1

Heger, A., Fryer, C. L., Woosley, S. E., Langer, N., \& Hartmann, D. H. 2003, ApJ, 591, 288

Hendry, M. A., Smartt, S. J., Crockett, R. M., Maund, J. R., Gal-Yam, A., Moon, D.-S., Cenko, S. B., Fox, D. W., Kudritzki, R. P., Benn, C. R., \& Østensen, R. 2006, MNRAS, 369, 1303

Li, W., Van Dyk, S. D., Filippenko, A. V., \& Cuillandre, J.-C. 2005, PASP, 117,121

Li, W., Van Dyk, S. D., Filippenko, A. V., Cuillandre, J.-C., Jha, S., Bloom, J. S., Riess, A. G., \& Livio, M. 2006, ApJ, 641, 1060

Li, W., Wang, X., Van Dyk, S. D., Cuillandre, J.-C., Foley, R. J., \& Filippenko, A. V. 2007, ApJ, 661, 1013

Marion, G. H., Kirshner, R., Wheeler, J. C., Vinko, J., Chornock, R., Foley,

R. J., Challis, P., Friedman, A., Garnavich, P., \& Krisciunas, K. 2011, The Astronomer's Telegram, 3435, 1

Maund, J. R., Fraser, M., Ergon, M., Pastorello, A., Smartt, S. J., Sollerman, J., Benetti, S., Botticella, M. ., Bufano, F., Danziger, I. J., Kotak, R., Magill, L., Stephens, A. W., \& Valenti, S. 2011, ArXiv e-prints

Maund, J. R., Smartt, S. J., \& Danziger, I. J. 2005, MNRAS, 364, L33

Schlegel, D. J., Finkbeiner, D. P., \& Davis, M. 1998, ApJ, 500, 525

Silverman, J. M., Filippenko, A. V., \& Cenko, S. B. 2011, The Astronomer's Telegram, 3398, 1

Smartt, S. J. 2009, ARA\&A, 47, 63

Smartt, S. J., Eldridge, J. J., Crockett, R. M., \& Maund, J. R. 2009, MNRAS, 395,1409

Smartt, S. J., Maund, J. R., Hendry, M. A., Tout, C. A., Gilmore, G. F., Mattila, S., \& Benn, C. R. 2004, Science, 303, 499

Smartt, S. J., Vreeswijk, P. M., Ramirez-Ruiz, E., Gilmore, G. F., Meikle, W. P. S., Ferguson, A. M. N., \& Knapen, J. H. 2002, ApJ, 572, L147

Smith, N., Li, W., Miller, A. A., Silverman, J. M., Filippenko, A. V., Cuillandre, J.-C., Cooper, M. C., Matheson, T., \& Van Dyk, S. D. 2011a, ApJ, 732, 63

Smith, N., Li, W., Silverman, J. M., Ganeshalingam, M., \& Filippenko, A. V. 2011b, MNRAS, 415, 773

Soderberg, A. M., Margutti, R., Zauderer, B. A., Krauss, M., Katz, B., Chomiuk, L., Dittmann, J. A., Nakar, E., Sakamoto, T., Kawai, N., Hurley, K., Barthelmy, S., Toizumi, T., Morii, M., Chevalier, R. A., Gurwell, M., Petitpas, G., Rupen, M., Alexander, K. D., Levesque, E. M., Fransson, C., Brunthaler, A., Bietenholz, M. F., Chugai, N., Connaughton, V., Briggs, M., Meegan, C., von Kienlin, A., Zhang, X., Rau, A., Golenetskii, S., Mazets, E., \& Cline, T. 2011, ArXiv e-prints

Tonry, J. L., Dressler, A., Blakeslee, J. P., Ajhar, E. A., Fletcher, A. B., Luppino, G. A., Metzger, M. R., \& Moore, C. B. 2001, ApJ, 546, 681

Van Dyk, S. D., Garnavich, P. M., Filippenko, A. V., Höflich, P., Kirshner, R. P., Kurucz, R. L., \& Challis, P. 2002, PASP, 114, 1322

Van Dyk, S. D., Li, W., Cenko, S. B., Kasliwal, M. M., Horesh, A., Ofek, E. O., Kraus, A. L., Silverman, J. M., Arcavi, I., Filippenko, A. V., Gal-Yam, A., Quimby, R. M., Kulkarni, S. R., Yaron, O., \& Polishook, D. 2011, ArXiv e-prints

Van Dyk, S. D., Li, W., \& Filippenko, A. V. 2003a, PASP, 115, 448 - 2003b, PASP, 115,1289

Van Dyk, S. D., Peng, C. Y., Barth, A. J., \& Filippenko, A. V. 1999, AJ, 118, 2331

Woosley, S. E., Heger, A., \& Weaver, T. A. 2002, Reviews of Modern Physics, 74, 1015 

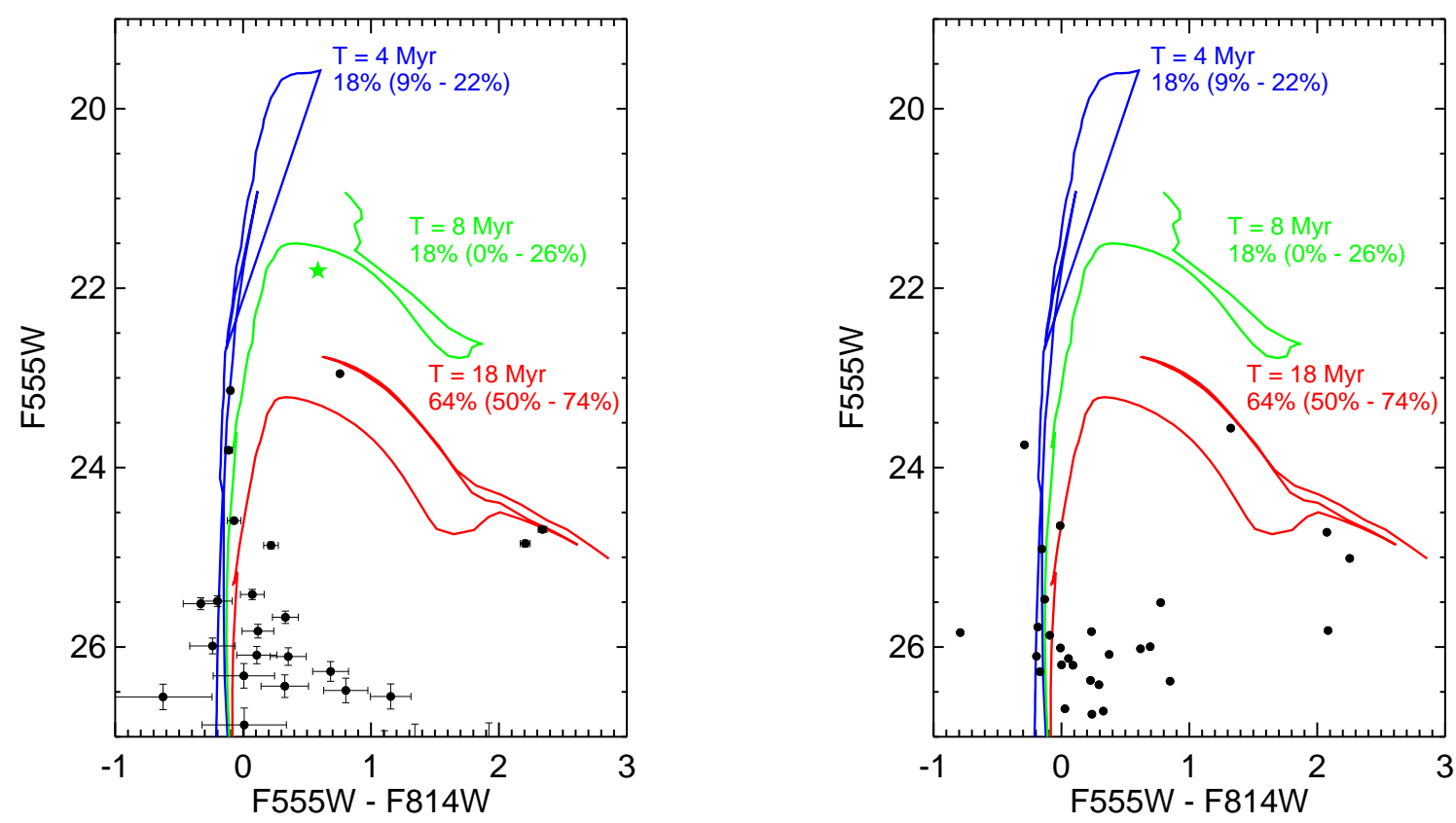

Figure 1. Observed (left panel) and modeled (right panel) color-magnitude diagrams (CMD). In the left panel, the brightest star (green star symbol) is the progenitor candidate (Maund et al. 2011; Van Dvk et al. 2011). The three lines correspond to Padova isochrones that are closest in age to the three SF bursts in Fig. 2 and each is labeled with the age and percent of total SF in the last $50 \mathrm{Myr}$. Note that we do not fit these isochrones to determine the age. Rather, we model the CMD and find the SFH that best represents the data. The right panel shows a representative CMD model from the MC simulations.
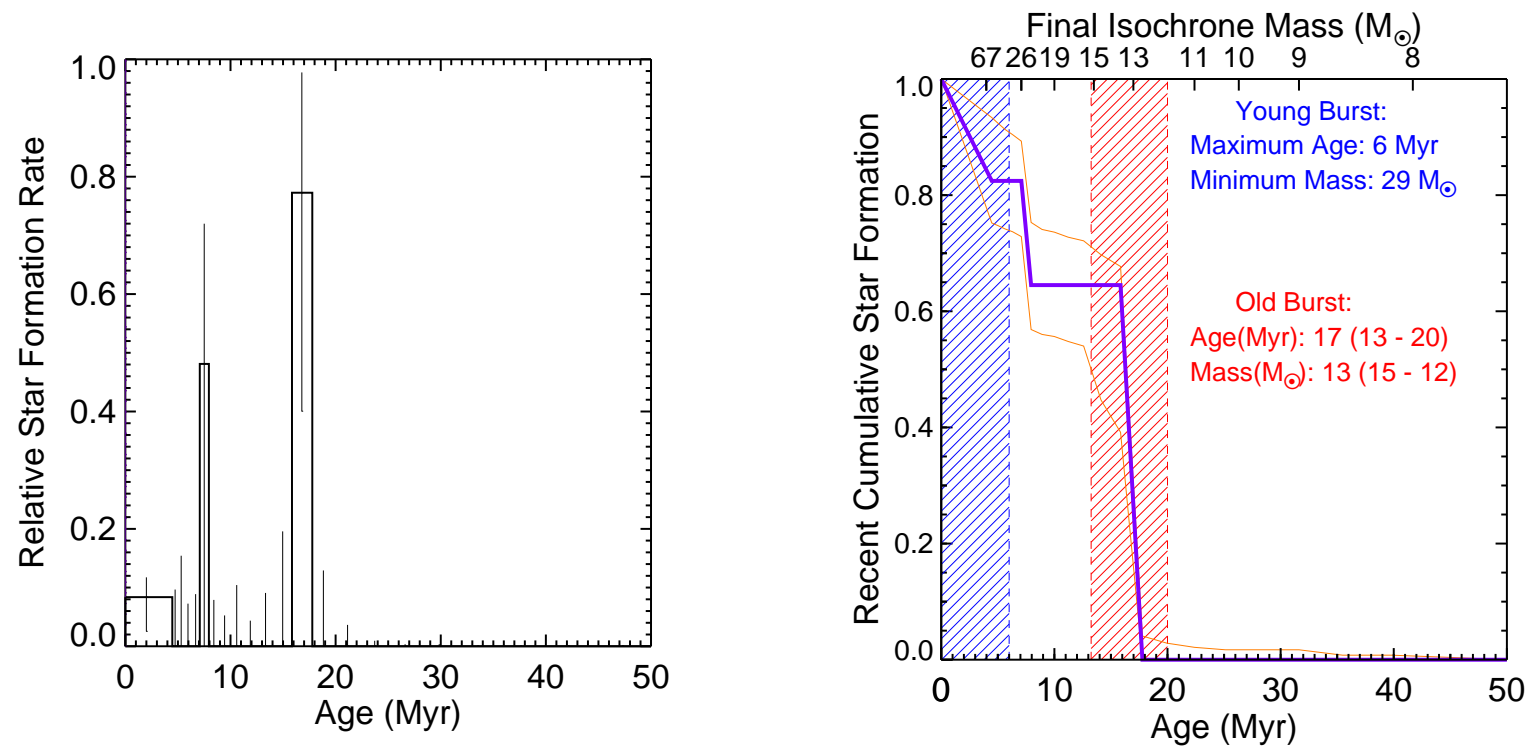

Figure 2. Modeled star formation history (SFH) in the vicinity of SN 2011dh. The left panel shows the relative star formation rate (solid black line). We use the cumulative SF since $50 \mathrm{Myr}$ ago (right panel) to characterize the age. We find two significant SF events at $<6 \mathrm{Myr}$ and $17_{-4}^{+3} \mathrm{Myr}$ (See $\S$ for a discussion of how we rule out the $8 \mathrm{Myr}$ old burst). The corresponding $M_{\text {ZAMS }}$ for the progenitor are $>29$ and $13_{-1}^{+2} \mathrm{M}_{\odot}$. Most of the recent SF occurred in the $17 \mathrm{Myr}$ burst. Therefore, our most probable $M_{\text {ZAMS }}$ estimate for the progenitor is $13_{-1}^{+2} \mathbf{M}_{\odot}$. 